\title{
Neuroanatomical correlates of perceiving the intensity and pleasantness of intense saltiness in healthy subjects
}

\author{
Andy Wai Kan Yeung \\ Oral and Maxillofacial Radiology, Applied Oral Sciences, Faculty of Dentistry, The University of Hong Kong, Hong Kong, China
}

\begin{abstract}
Reducing excessive dietary salt is beneficial to human health. Various taste-related brain regions are activated upon taste reception. However, it is unknown if their structural volumes would also influence taste perception. This study aimed to examine if grey matter volume would correlate with intensity or pleasantness scores given by participants after tasting an intense salty taste solution. We recruited 34 healthy human adults. They tasted $2 \mathrm{ml}$ of $0.5 \mathrm{M}$ sodium chloride solution and underwent magnetic resonance imaging to record their brain structures. By voxelbased morphometry, the amygdala, thalamus, insula and orbitofrontal cortex were structurally correlated to tasting.
\end{abstract}

Keywords: Grey matter volume, human health, magnetic resonance imaging, salty taste, voxel-based morphometry.

THERE is substantial evidence that reducing excessive dietary salt intake is beneficial to human health by reducing blood pressure and the risk of cardiovascular diseases ${ }^{1,2}$. We need to understand how our brains process intense salty taste to devise better strategies to reduce excessive salt consumption, such as the use of glutathione to augment salty and umami tastes ${ }^{3}$.

Neuroimaging studies have comprehensively reported on various aspects of brain responses to salty taste stimulations. When sodium chloride $(\mathrm{NaCl})$ solutions were applied to human tongues, the brains of the participants were commonly activated at various regions such as the thalamus, insula, orbitofrontal cortex (OFC) and amygda$1 \mathrm{a}^{4-8}$. In particular, activity in the insula and amygdala is modulated by the concentration of $\mathrm{NaCl}$ solution ${ }^{6,9}$. However, to the best of my knowledge, there is no published information regarding the neuroanatomical correlates of individual variations in taste perception among the healthy population. Appropriate training and exposure to visual perceptual acuity or complex motor skill tasks have been found to induce structural changes in the relevant brain regions ${ }^{10,11}$. This effect might potentially be utilized to assess or facilitate the reduction of excessive dietary salt consumption if the grey matter volume in

e-mail: ndyeung@hku.hk taste-related regions are found to correlate with the perceived intensity of salty taste.

By linking behavioural scores with quantitative assessments of neural tissue, particularly grey matter volume, the neuroanatomical basis of chemosensory perception could be established, and such associations could possibly be utilized as biomarkers. Voxel-based morphometry (VBM) is a standard and automated method used to segment the brain into grey matter, white matter and cerebrospinal fluid volumes ${ }^{12}$. The voxel-wise grey matter volume obtained from each participant reflects individual anatomical variabilities and could be used to assess correlations with behavioural or performance scores. Unlike taste studies, there have been numerous smell studies that have revealed a positive correlation between grey matter volume in the olfactory cortices and olfactory bulb, and olfactory performance among healthy participants $^{13,14}$. Additionally, there is a reduction in grey matter volume in the olfactory cortices in patients who have lost the sense of smell ${ }^{15,16}$. As taste is a chemosensory perception similar to smell, such correlations may also be valid for taste.

Therefore, the aim of this study was to examine if there are neuroanatomical correlates of intense salty taste perception. Naturally, there are inter-individual variations in the perception of the intensity and pleasantness aspects of an intense salty taste. The present author hypothesized that such variations would positively correlate with grey matter volume in the relevant salty taste-related brain regions, namely the thalamus, insula, OFC and amygdala.

\section{Materials and methods}

\section{Participants}

The participants were recruited through The University of Hong Kong community. Thirty-four healthy adults (17 males and 17 females) participated in this study. Their mean age was 21.3 years $(\mathrm{SD}=2.4)$, and their mean body mass index (BMI) was $20.2 \mathrm{~kg} / \mathrm{m}^{2}(\mathrm{SD}=2.1)$. All participants were right-handed, with a mean Edinburgh Handedness Inventory ${ }^{17}$ score of $74.6(\mathrm{SD}=20.4)$. None of them reported a history of psychiatric or neurological 


\section{RESEARCH ARTICLES}

deficits, brain injuries, substance abuse or psychiatric medication. People were not recruited into the study if they were obese, smokers, or currently taking medication, or if they had any known taste or smell disorders, active oral lesions, or any MRI exclusion criteria (e.g. claustrophobia, history of back pain, or metal devices such as a pacemaker). This study adhered to the Declaration of Helsinki for Medical Research involving Human Subjects. The Institutional Review Board of The University of Hong Kong/Hospital Authority Hong Kong West Cluster approved the study, and all participants provided written informed consent. The analysis of brain functions of these participants has been published elsewhere ${ }^{6}$. In this article, an analysis of their brain structures is reported.

\section{Assessment of intensity and pleasantness of a salty solution}

The participants received a bolus of $2 \mathrm{ml}$ of $0.5 \mathrm{M} \mathrm{NaCl}$ solution held in a polypropylene cup without knowing the contents. The concentration of $0.5 \mathrm{M}$ was chosen because this is equivalent to the salt content in ocean water, which humans normally perceive as intense and unpleasant ${ }^{9,18}$. Moreover, since the neuroanatomical correlates of taste intensity and pleasantness have not been elucidated before, it is perhaps most suitable to begin with a strong stimulus. Each participant was instructed to pour the entire contents of the cup into his/her mouth, hold it without gargling or swallowing, and spit it out after $3 \mathrm{sec}$. Participants rated the intensity and pleasantness of the solution on a $0-10$ visual analog scale (VAS; 0 was labelled 'not intense/pleasant at all', and 10 as 'strongest intensity/ pleasantness imaginable'). The participants then immediately underwent magnetic resonance imaging (MRI) scanning to obtain structural data of their brains. The results from their functional scans have been reported elsewhere ${ }^{6}$.

An average score significantly different from the midpoint of the VAS scale would imply that the solution was not generally perceived as neutral by the participants ${ }^{4,19}$. To confirm this, the intensity and pleasantness scores rated by the participants were entered into SPSS 23.0 (IBM, New York, USA) and one-sample $t$-tests were performed. Test results with $P<0.05$ were considered statistically significant.

\section{MRI data acquisition}

All MRI data were obtained at the MRI Unit of the Department of Diagnostic Radiology at The University of Hong Kong. Participants were scanned with a 3-Tesla scanner (Philips Achieva 3.0 System; Philips Medical System, The Netherlands) using a standard receiving eight-channel head coil and the same parameters. Highresolution T1-weighted images of the brain were acquired using a magnetization-prepared rapid gradient-echo (MPRAGE) sequence (repetition time $=6.909 \mathrm{~ms}$, echo time $=3.147 \mathrm{~ms}, \quad$ flip angle $=8^{\circ}$, field of view $=$ $250 \mathrm{~mm} \times 250 \mathrm{~mm}$, voxel size $=1 \mathrm{~mm} \times 1 \mathrm{~mm} \times 1 \mathrm{~mm}$, slice thickness $=1 \mathrm{~mm}, 155$ slices, total acquisition time $=9: 56 \mathrm{~min})$.

\section{MRI data preprocessing}

Data were processed and examined with SPM8 (Wellcome Trust Centre for Neuroimaging, University College of London, London, UK) and VBM8 (revision 435) toolbox (Department of Psychiatry, University of Jena, Germany) under a MATLAB R2012a space (The Mathworks Inc, Natick, MA, USA) running on an OS X Mavericks computer. Standard procedures and default parameters of the VBM8 toolbox were used as reported in the literature $^{15,16}$ and are described briefly herein. The images were bias-corrected, pre-registered to standardized Montréal Neurological Institute (MNI) space and estimated using the DARTEL algorithm ${ }^{20}$, and tissues were classified into grey matter, white matter and cerebrospinal fluid segments in voxel size of $1.5 \mathrm{~mm} \times$ $1.5 \mathrm{~mm} \times 1.5 \mathrm{~mm}$. Grey matter segments had their voxel values multiplied (modulated) by the nonlinear components derived from the normalization matrix. This modulation allowed a comparison of the absolute amount of grey matter corrected for individual brain sizes. Finally, modulated grey matter segments were smoothed with a $12 \mathrm{~mm}$ full-width at half-maximum isotropic Gaussian kernel. This workflow ensured that the brain images of each participant were properly normalized for comparison.

\section{Statistical analysis}

The voxel-wise correlation between grey matter volume of the participants and their corresponding intensity/pleasantness scores for salty solution was tested using linear regression models. As the participants had a balanced gender composition and were relatively homogeneous in age and BMI, these factors were not entered into the model as covariates. To avoid possible edge effects between different tissue types, an absolute threshold masking was applied by excluding all voxels with grey matter values of less than 0.1 (ref. 15). Region-of-interest (ROI) analysis was performed in taste-related regions, like the thalamus, insula, OFC and amygdala because they are relevant to salty taste processing, including intensity and pleasantness ${ }^{4,9,21}$. The Wake Forest University (WFU) Pickatlas was used to develop a mask containing all the above-mentioned regions in both hemispheres ${ }^{22-24}$. A previous study on the association between grey matter volume and smell (another component of chemosensory perception in addition to taste) applied a threshold of 
$P<0.01$ across the whole brain ${ }^{15}$. The protocol of a more recent study that applied a threshold of uncorrected $P<0.01$ with a minimum cluster size of 100 voxels was followed ${ }^{16}$.

For illustrative purposes, data from peak voxels were extracted to form scatter plots with Pearson's $r$ and regression equations. All statistical analyses were performed with SPSS 23.0 (IBM, New York, USA) with the significance level set at 0.05 .

\section{Results}

Thirty-four subjects were recruited for this study, and there were no dropouts or missing data for the whole experiment.

\section{Behavioural score results}

The mean intensity score was $8.00(\mathrm{SD}=1.60)$, which was significantly higher than the midpoint of VAS $(t=10.92, P<0.001)$. The mean pleasantness score was $2.56(\mathrm{SD}=1.95)$, which was significantly lower than the midpoint of VAS $(t=-7.30, P<0.001)$.

These scores confirmed that participants in general perceived the solution as intense and unpleasant. Moreover, the intensity and pleasantness scores demonstrated a negative correlation $(r=-0.55, P=0.001$, Figure 1$)$.

\section{Correlation between grey matter volume and intensity score}

There was a positive correlation between intensity scores and grey matter volumes of the participants in the thalamus, middle dorsal insula and OFC in the left hemisphere (Table 1 and Figure 2). The voxels showing correlation

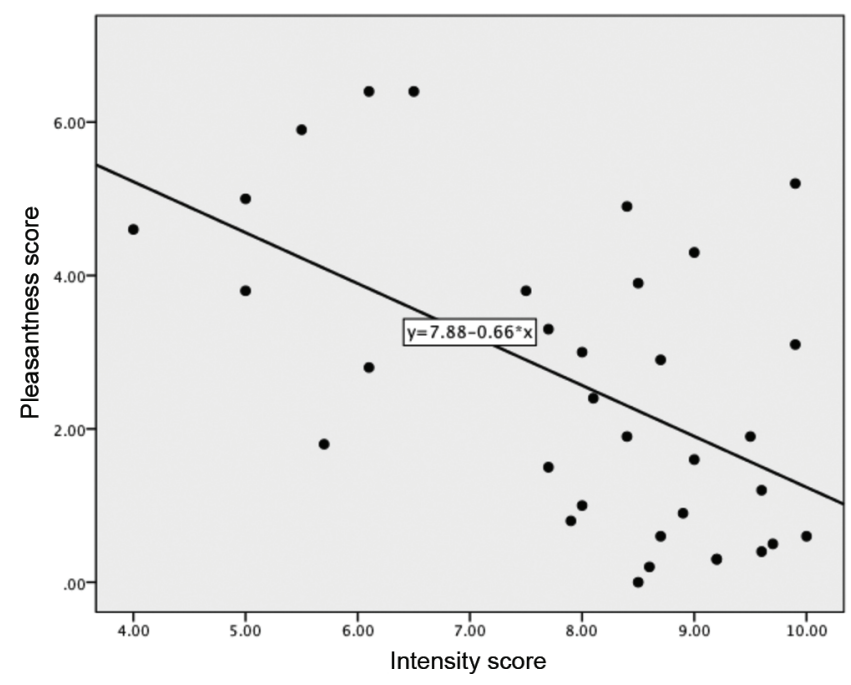

Figure 1. Scatter plot of the intensity and pleasantness scores. did not strictly correspond to the activated voxels only. There were no significant negative correlations detected.

\section{Correlation between grey matter volume and pleasantness score}

There was a positive correlation between pleasantness scores and grey matter volumes of the participants in the right amygdala (Table 1 and Figure 3 ). There were no significant negative correlations detected.

\section{Discussion}

The thalamus and insula have been reported to be functionally relevant to salty taste intensity in healthy participants, as their activations are modulated by salty taste intensity $^{6,9}$. With the same group of participants as that from a study by Yeung et al. ${ }^{6}$, the present analysis reveals that the thalamus and insula also have a structural correlate with salty taste intensity. In the past, it has been reported that patients with lesions in their insula had a reduction in the perception of saltiness compared to healthy counterparts ${ }^{25,26}$. The present study demonstrates such neuroanatomical correlates of intense saltiness perception in the insula of healthy participants. The results of the study show that a larger grey matter volume in the insula is associated with a higher perceived intensity. The grey matter volume in the thalamus also shows similar positive correlation. These results are reasonable because a larger grey matter volume is equivalent to having more neural cell bodies and, thus, potentially a higher chance of having more taste-responsive neural cells within the relevant regions. Hence, these regions might have stronger responses to taste stimulations.

It has been reported that the amygdala is activated in response to salty taste stimulations that are perceived as aversive or unpleasant ${ }^{4,5}$; similarly, the amygdala is also activated in response to pleasant tastes or other stimulations ${ }^{4,27}$. Results from this study reveal a correlation between grey matter volume in the amygdala and pleasantness score of a $\mathrm{NaCl}$ solution. This confirms that the amygdala is an important structure that has both functional and structural relevance to the perception of taste pleasantness. Previous studies have demonstrated that smaller grey matter volumes in the amygdala are associated with a wide range of negative/unfavourable emotions such as neuroticism (a personality trait with a long-term tendency for a negative mood) and depression ${ }^{28,29}$. In the present study, participants with smaller grey matter volumes in their amygdala perceived the $\mathrm{NaCl}$ solution as more unpleasant. This correlation is consistent with previous reports ${ }^{28,29}$. Since excessive salt intake may lead to high blood pressure and cardiovascular diseases ${ }^{1}$, future studies may recruit these patients and compare their grey 

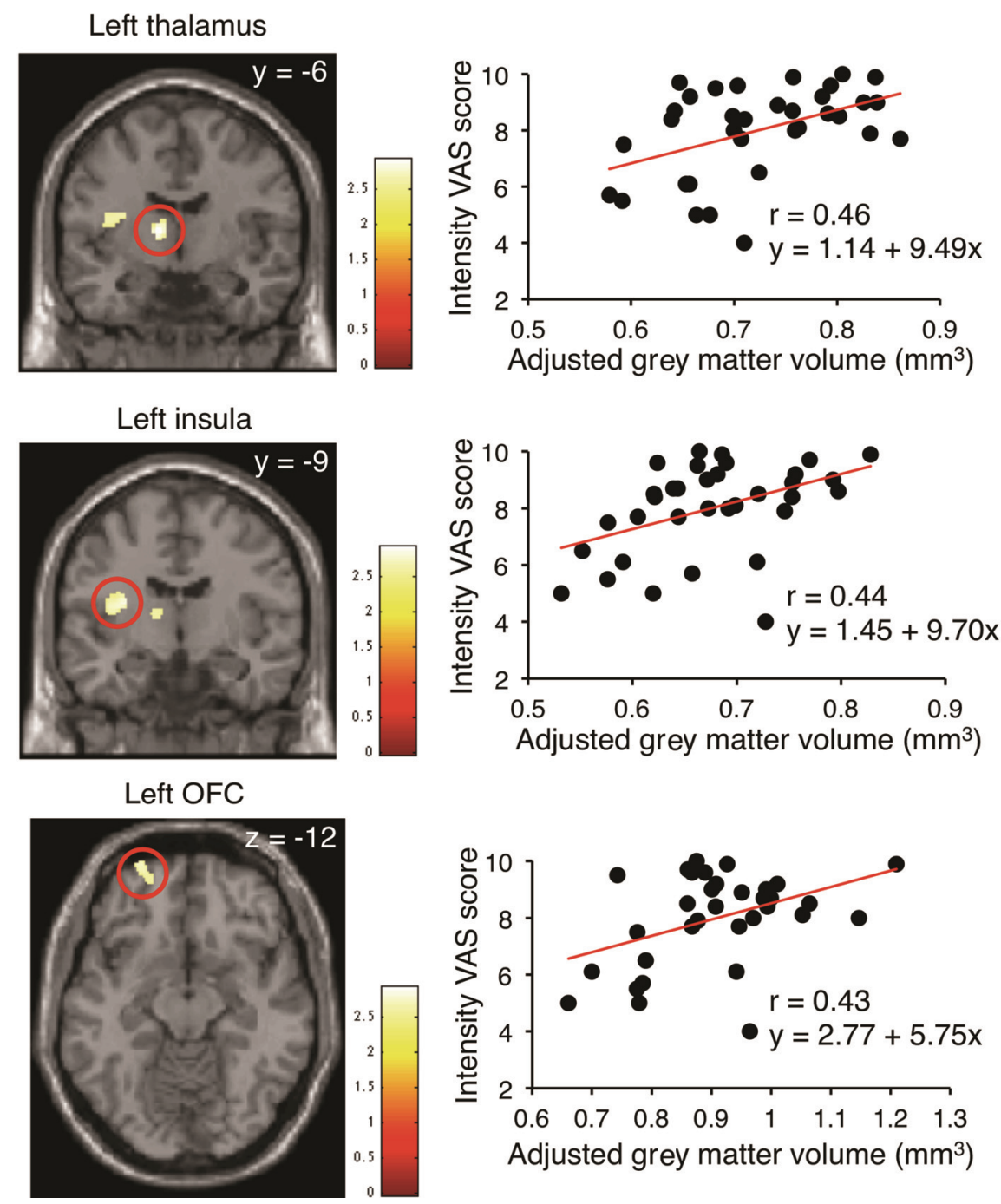

Figure 2. Correlation of grey matter volume in various brain regions to the intensity score given by the participants. Results show that the grey matter volume in the left thalamus, insula and OFC is positively correlated to the intensity score. The images are thresholded at $P<0.01$ with a minimum cluster size of 100 voxels, while the colour bar depicts the range of $t$ values of the significant voxels. The scatter plots illustrate significant correlation of grey matter volume of the peak voxel to intensity score with Pearson's $r$ and regression formula. The adjusted grey matter volume is calculated by multiplying the voxel size $\left(3.375 \mathrm{~mm}^{3}\right)$ by the percentage of grey matter present in the voxel calculated by VBM8 with partial volume segmentation and corrected for individual differences in brain size. OFC, Orbitofrontal cortex. VAS, Visual analog scale.

matter volume in the amygdala with a healthy control group.

It is interesting to find a correlation between grey matter volume in the OFC and taste intensity but not with pleasantness score. The OFC is known to represent or process taste pleasantness ${ }^{30,31}$. While it has been reported that the responses in the OFC were positively correlated with the pleasantness ratings of sucrose tastes and strawberry smells $^{30}$, no such correlations were reported for salty taste pleasantness. Previous studies have only reported activation in the OFC when receiving a salty taste without mentioning its correlation with intensity or pleasantness scores ${ }^{4,5,32}$. Spetter et al. ${ }^{9}$ reported a correlation between brain responses to salty tastes and corres- ponding intensity scores, but the OFC was not among the regions examined. Hence, the results reported in this study suggest that there might be complex processing of salty taste perception in the OFC, such that the structural correlates of salty taste intensity might contribute to its activation relative to taste pleasantness.

There are some limitations in this study. First, the sample size is only 34 , which is relatively small. This sample size is comparable to those reported in VBM studies related to smell ${ }^{15,16}$, but a larger sample size would further increase the statistical power. Second, only intense salty taste has been tested. The neuroanatomical correlates of taste intensity and pleasantness perception established in this study may not be extended to other 
Table 1. Brain regions showing a correlation between grey matter volume and intensity or pleasantness score

\begin{tabular}{|c|c|c|c|c|c|c|c|}
\hline \multirow[b]{2}{*}{ Region } & \multirow[b]{2}{*}{$\operatorname{Side}^{\mathrm{a}}$} & \multicolumn{3}{|c|}{ MNI coordinates } & \multirow[b]{2}{*}{$t$ score } & \multirow[b]{2}{*}{ Cluster size (voxels) } & \multirow[b]{2}{*}{$P$} \\
\hline & & $x$ & $y$ & $z$ & & & \\
\hline \multicolumn{8}{|l|}{ Intensity } \\
\hline \multicolumn{8}{|c|}{ Positive correlation } \\
\hline Thalamus & $\mathrm{L}$ & -10 & -6 & 7 & 2.92 & 113 & 0.003 \\
\hline Insula & $\mathrm{L}$ & -30 & -9 & 16 & 2.78 & 164 & 0.004 \\
\hline OFC & $\mathrm{L}$ & -26 & 63 & -12 & 2.69 & 140 & 0.006 \\
\hline \multicolumn{7}{|c|}{ Negative correlation } & \\
\hline \multicolumn{8}{|l|}{ Pleasantness } \\
\hline \multicolumn{8}{|c|}{ Positive correlation } \\
\hline Amygdala & $\mathrm{R}$ & 21 & 0 & -24 & 3.19 & 102 & 0.002 \\
\hline $\begin{array}{l}\text { Negative corr } \\
\text { Not signific }\end{array}$ & & & & & & & \\
\hline
\end{tabular}

The result is thresholded at $P<0.01$ (uncorrected) with a minimum cluster size of 100 voxels. All coordinates are given in Montreal Neurological Institute (MNI) space. $P$ values from peak voxels are reported. Significant correlation with intensity score is not found on the right side of the brain, whereas significant correlation with pleasantness score is not found on the left side of the brain. ${ }^{a} \mathrm{~L}$, Left; R, right.
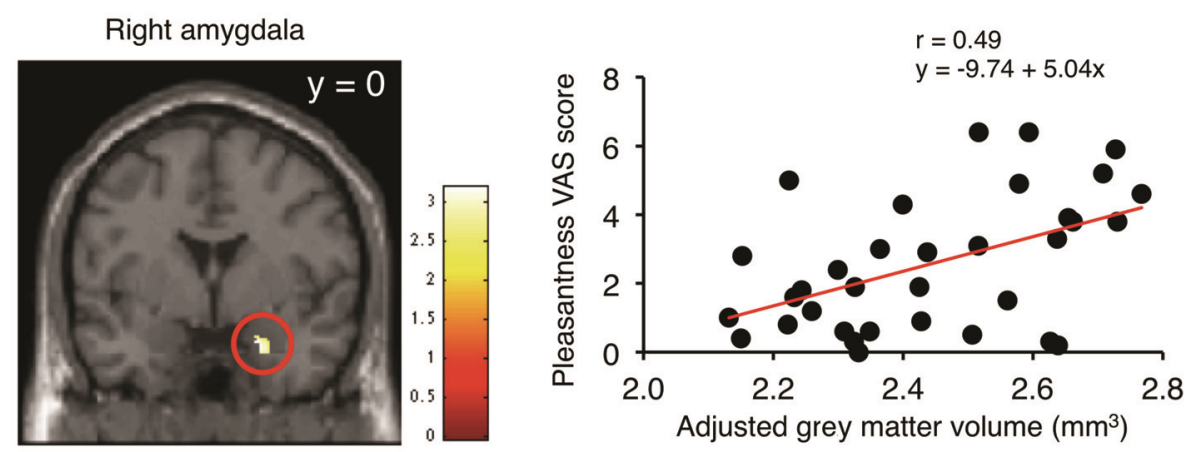

Figure 3. Correlation of grey matter volume in the right amygdala to the pleasantness score given by the participants. Results show that the grey matter volume in the right amygdala is positively correlated to the pleasantness score. The image is thresholded at $P<0.01$ with a minimum cluster size of 100 voxels, while the colour bar depicts the range of $t$ values of the significant voxels. The scatter plot illustrates the significant correlation of grey matter volume of peak voxel to the pleasantness score with Pearson's $r$ and regression formula. The adjusted grey matter volume is calculated by multiplying the voxel size $\left(3.375 \mathrm{~mm}^{3}\right)$ by the percentage of grey matter present in the voxel calculated by VBM8 with partial volume segmentation and corrected for individual differences in brain size.

tastes such as sweet, sour, bitter and umami or weak tastes because their underlying intensity and pleasantness profiles may be different. For instance, low-intensity sweet taste can be pleasant but low-intensity bitter taste is unpleasant. However, it is worth mentioning that sweet and salty taste intensity processing seems to converge in the insula ${ }^{9}$ and that there is no different processing of taste concentration across sweet, sour, salty and bitter solutions in the insula ${ }^{33}$.

\section{Conclusion}

This study demonstrate that the grey matter volumes in the left thalamus, insula and OFC were positively corre- lated with intensity scores given to an intense salty taste among healthy, right-handed, young adults. The grey matter volume in the right amygdala was positively correlated to pleasantness scores. The study provides insight into the neuroanatomical correlates of taste intensity and pleasantness perceptions among healthy participants, therefore implying that these regions have not only functional but also structural relevance to taste sensations. Future studies should further examine the associations between structural volume of taste-related brain regions and the corresponding taste perceptions.

Conflict of interest: The author declares no conflict of interest. 
1. Aburto, N. J., Ziolkovska, A., Hooper, L., Elliott, P., Cappuccio, F. P. and Meerpohl, J. J., Effect of lower sodium intake on health: systematic review and meta-analyses. Br. Med. J., 2013, 346, f1326.

2. Mozaffarian, D. et al., Global sodium consumption and death from cardiovascular causes. N. Engl. J. Med., 2014, 371, 624-634.

3. Goto, T. K., Yeung, A. W. K., Tanabe, H. C., Ito, Y., Jung, H.-S. and Ninomiya, Y., Enhancement of combined umami and salty taste by glutathione in the human tongue and brain. Chem. Senses, 2016, 41, 623-630.

4. O'Doherty, J., Rolls, E. T., Francis, S., Bowtell, R. and McGlone, F., Representation of pleasant and aversive taste in the human brain. J. Neurophysiol., 2001, 85, 1315-1321.

5. Zald, D. H., Lee, J. T., Fluegel, K. W. and Pardo, J. V., Aversive gustatory stimulation activates limbic circuits in humans. Brain, 1998, 121, 1143-1154.

6. Yeung, A. W. K., Tanabe, H. C., Suen, J. L. K. and Goto, T. K., Taste intensity modulates effective connectivity from the insular cortex to the thalamus in humans. Neuroimage, 2016, 135, 214 222

7. Yeung, A. W. K., Goto, T. K. and Leung, W. K., Basic taste processing recruits bilateral anteroventral and middle dorsal insulae: an activation likelihood estimation meta-analysis of fMRI studies. Brain Behav., 2017, 7, e00655.

8. Yeung, A. W. K., Goto, T. K. and Leung, W. K., Affective value, intensity and quality of liquid tastants/food discernment in the human brain: an activation likelihood estimation meta-analysis. Neuroimage, 2018, 169, 189-199.

9. Spetter, M., Smeets, P., de Graaf, C. and Viergever, M., Representation of sweet and salty taste intensity in the brain. Chem. Senses, 2010, 35, 831-840.

10. Bezzola, L., Mérillat, S., Gaser, C. and Jäncke, L., Traininginduced neural plasticity in golf novices. J. Neurosci., 2011, 31, 12444-12448.

11. Maguire, E. A., Gadian, D. G., Johnsrude, I. S., Good, C. D., Ashburner, J., Frackowiak, R. S. and Frith, C. D., Navigation-related structural change in the hippocampi of taxi drivers. Proc. Natl. Acad. Sci. USA, 2000, 97, 4398-4403.

12. Ashburner, J. and Friston, K. J., Voxel-based morphometry - the methods. Neuroimage, 2000, 11, 805-821.

13. Seubert, J., Freiherr, J., Frasnelli, J., Hummel, T. and Lundström, J. N., Orbitofrontal cortex and olfactory bulb volume predict distinct aspects of olfactory performance in healthy subjects. Cereb. Cortex, 2013, 23, 2448-2456.

14. Frasnelli, J., Lundström, J. N., Boyle, J. A., Djordjevic, J., Zatorre, R. J. and Jones-Gotman, M., Neuroanatomical correlates of olfactory performance. Exp. Brain Res., 2010, 201, 1-11.

15. Bitter, T., Gudziol, H., Burmeister, H. P., Mentzel, H.-J., Guntinas-Lichius, O. and Gaser, C., Anosmia leads to a loss of gray matter in cortical brain areas. Chem. Senses, 2010, 35, 407-415.

16. Yao, L., Pinto, J. M., Yi, X., Li, L., Peng, P. and Wei, Y., Gray matter volume reduction of olfactory cortices in patients with idiopathic olfactory loss. Chem. Senses, 2014, bju047.

17. Oldfield, R. C., The assessment and analysis of handedness: the Edinburgh inventory. Neuropsychologia, 1971, 9, 97-113.

18. Bolhuis, D. P., Lakemond, C. M., de Wijk, R. A., Luning, P. A. and de Graaf, C., Effect of salt intensity on ad libitum intake of tomato soup similar in palatability and on salt preference after consumption. Chem. Senses, 2010, 35, 789-799.
19. Small, D. M., Gregory, M. D., Mak, Y. E., Gitelman, D., Mesulam, M. M. and Parrish, T., Dissociation of neural representation of intensity and affective valuation in human gustation. Neuron, 2003, 39, 701-711.

20. Ashburner, J., A fast diffeomorphic image registration algorithm. Neuroimage, 2007, 38, 95-113.

21. Iannilli, E., Singh, P. B., Schuster, B., Gerber, J. and Hummel, T., Taste laterality studied by means of umami and salt stimuli: an fMRI study. Neuroimage, 2012, 60, 426-435.

22. Maldjian, J. A., Laurienti, P. J. and Burdette, J. H., Precentral gyrus discrepancy in electronic versions of the Talairach atlas. Neuroimage, 2004, 21, 450-455.

23. Maldjian, J. A., Laurienti, P. J., Kraft, R. A. and Burdette, J. H., An automated method for neuroanatomic and cytoarchitectonic atlas-based interrogation of fMRI data sets. Neuroimage, 2003, 19, $1233-1239$

24. Tzourio-Mazoyer, N. et al., Automated anatomical labeling of activations in SPM using a macroscopic anatomical parcellation of the MNI MRI single-subject brain. Neuroimage, 2002, 15, 273289.

25. Mak, Y. E., Simmons, K. B., Gitelman, D. R. and Small, D. M., Taste and olfactory intensity perception changes following left insular stroke. Behav. Neurosci., 2005, 119, 1693.

26. Pritchard, T. C., Macaluso, D. A. and Eslinger, P. J., Taste perception in patients with insular cortex lesions. Behav. Neurosci., 1999, 113, 663.

27. Zald, D. H., The human amygdala and the emotional evaluation of sensory stimuli. Brain Res. Rev., 2003, 41, 88-123.

28. Omura, K., Constable, R. T. and Canli, T., Amygdala gray matter concentration is associated with extraversion and neuroticism. Neuroreport., 2005, 16, 1905-1908.

29. Pezawas, L. et al., 5-HTTLPR polymorphism impacts human cingulate-amygdala interactions: a genetic susceptibility mechanism for depression. Nature Neurosci., 2005, 8, 828-834.

30. De Araujo, I. E., Rolls, E. T., Kringelbach, M. L., McGlone, F. and Phillips, N., Taste-olfactory convergence, and the representation of the pleasantness of flavour, in the human brain. Eur. J. Neurosci., 2003, 18, 2059-2068.

31. Small, D. M., Bender, G., Veldhuizen, M. G., Rudenga, K., Nachtigal, D. and Felsted, J., The role of the human orbitofrontal cortex in taste and flavor processing. Ann. N.Y. Acad. Sci., 2007, 1121, 136-151.

32. O’Doherty, J. P., Deichmann, R., Critchley, H. D. and Dolan, R. J., Neural responses during anticipation of a primary taste reward. Neuron, 2002, 33, 815-826.

33. Dalenberg, J. R., Hoogeveen, H. R., Renken, R. J., Langers, D. R. and ter Horst, G. J., Functional specialization of the male insula during taste perception. Neuroimage, 2015, 119, 210-220.

ACKNOWLEDGEMENTS. This paper is a re-analysis of existing data, and hence did not receive any funding. I thank Dr Florian Kurth, Department of Neurology, UCLA Brain Mapping Center, USA, for clarifying concepts and assisting in setting the VBM8 procedures for the analyses reported in this study.

Received 20 February 2018; revised accepted 1 October 2018

doi: $10.18520 / \mathrm{cs} / \mathrm{v} 116 / \mathrm{i} 1 / 69-74$ 